\title{
Asymmetric Social Norms ${ }^{\dagger}$
}

\author{
Gabriele Camera Alessandro Gioffré \\ Chapman University Goethe University \\ University of Basel
}

December 8, 2016

\begin{abstract}
Studies of cooperation in infinitely repeated matching games focus on homogeneous economies, where full cooperation is efficient and any defection is collectively sanctioned. Here we study heterogeneous economies where occasional defections are part of efficient play, and show how to support those outcomes through contagious punishments.

Keywords: cooperation, repeated games, social dilemmas.

JEL codes: C6, C7

\footnotetext{
'We thank an anonymous referee for comments that improved the exposition of the paper. Partial support from NSF grant CCF-1101627 is acknowledged. Camera: Economic Science Institute, Chapman University, One University dr., Orange, CA 92866; camera@chapman.edu. Gioffré: Research Center SAFE, Goethe University Frankfurt, Theodor-W.-Adorno-Platz 3, 60629 Frankfurt; gioffre@safe.uni-frankfurt.de.
} 


\section{Introduction}

The social norms literature has extended the study of cooperation in infinitely repeated games from the case of stable partnerships (Rubinstein, 1979; Fudenberg and Maskin, 1986) to unstable meetings among homogeneous strangers (Kandori, 1992; Ellison, 1994). Patient strangers can attain the efficient outcome by triggering community-wide responses to privately observed defections ("grim" play). However, this requires sufficiently small groups. Large groups must be able to publicly monitor defections, which makes the economy's size irrelevant, and homogeneity greatly reduces the information that must be shared; since full cooperation is efficient, knowing that not everyone acted identically is sufficient. The open question is how results change when strangers are heterogeneous. Here, the structure of incentives may vary across meetings and efficient play may require some players to cooperate and others not.

We study social norms among heterogeneous strangers. Players receive iid productivity shocks, so payoff matrices stochastically vary across meetings, and can be asymmetric. Before choosing an action, players see productivities in their match. If full cooperation is efficient, publicly exposing defections supports cooperation; heterogeneity simply alters the admissible discount factors relative to the homogeneous case. Otherwise, if occasional defections are part of efficient play, then we need contagious punishments to support high payoffs because publicly exposing defections without productivities in other matches cannot reveal off-equilibrium play. Contagious punishment can deter defections only under moderate productivity differences.

The analysis has merit because little exists about cooperation under heterogeneity. The closest paper is Blonski and Spagnolo (2015), an infinitely repeated PD game in fixed pairs where cooperation is efficient but asymmetrically benefits players. The technique we present generates tractable closed-form expressions for continuation payoffs, which can be employed to calibrate laboratory economies. 


\section{Model}

In every period $N \geq 4$ (even) players are paired with uniform probability (Kandori, 1992; Ellison, 1994). Subsequently, each player $i=1, \ldots, N$, draws a random iid productivity shock $\theta_{i} \in\{1, \alpha\}, \alpha>1: \theta_{i}=\alpha$ (productive) with probability $q$, and $\theta_{i}=1$ (unproductive) otherwise. A match between $i$ and opponent $-i$ is either symmetric $\left(\theta_{i}=\theta_{-i}\right)$ or asymmetric. Payoffs are in Figure 1.

\begin{tabular}{cc|c|c|}
\multicolumn{4}{c}{ Player $-i$} \\
\cline { 3 - 4 } Player $i$ & $C$ & $\theta_{i} c, \theta_{-i} c$ & $D$ \\
\cline { 3 - 4 } & $D$ & $\theta_{-i}(c+g),-l, \theta_{i}(c+g)$ \\
\cline { 3 - 4 } & &
\end{tabular}

Figure 1: The game between player $i$ and $-i$.

Let $c, g, l>0$ and $\theta_{i} c>\theta_{i} g-l$. The cooperative outcome $(C, C)$ maximizes total earnings if

$$
c \geq \alpha g-l
$$

thus amounting to a proper Prisoners' Dilemma (PD). Otherwise, we have an asymmetric social dilemma where $D$ is dominant but asymmetric cooperation, $(D, C)$ or $(C, D)$, is efficient. Iterated PDs in fixed pairs assume (1) to rule out taking turns at selecting $C$ and $D$ (e.g., Rapoport and Chammah, 1965). Interestingly, Kandori (1992) and Ellison (1994) do not assume (1), possibly because pairs are short-lived and break up over time, which complicates coordination on action alternation.

Before choosing, players observe productivities only in their match, not in others. At the period's end players are informed if everyone chose identically or not. Full defection is an equilibrium - giving payoff 0 -because $D$ is a best response to $D$ by everyone else. Other equilibria exist. If (1) holds, public monitoring supports full cooperation. Everyone chooses $C$ unless someone acted differently, in which case everyone chooses $D$ forever. This equilibrium exists-independent 
of $N$-if

$$
\beta \geq \frac{c(\alpha-1)+g \alpha}{q c(\alpha-1)+\alpha(c+g)} \in(0,1) .
$$

If (1) does not hold, the available public information is no longer useful to attain efficiency. Earnings in matches with unequal productivities are maximized by asymmetric cooperation; hence, $(C, D)$ is part of efficient play. As players cannot see productivities in other matches, making defections public cannot reveal deviations from efficient play.

Hence, consider community-based enforcement triggered by privately observed deviations. Players cooperate whenever their productivity is no smaller than their opponent's; otherwise, they defect. If they observe someone choosing $D$ when $C$ should be chosen, then they switch to play "always defect."

Definition 1 (Asymmetric cooperation). At the start of any period, player $i$ either (i) "cooperates" by choosing $C$ if $\theta_{i} \geq \theta_{-i}$, and $D$ otherwise, or (ii) "punishes" by unconditionally choosing D. Player $i$ follows "cooperate" but permanently switches to "punish" if someone deviates to $D$ when $C$ should be chosen.

The equilibrium payoff is $(1-\beta)^{-1} \pi^{*}$, where

$$
\pi^{*}=q^{2} \alpha c+(1-q)^{2} c+(1-q) q(\alpha c+\alpha g-l)
$$

denotes expected period earnings.

Theorem 1. Fix q. If $\frac{c}{g}$ is sufficiently small, then there exists $\alpha \in\left(\underline{\alpha}, \alpha_{q}\right)$ such that if $\beta$ and $l$ are sufficiently large, then asymmetric cooperation is a sequential equilibrium.

The conditions on discounting $\beta$ and sucker's payoff $l$ are standard. Players must be patient to prefer $C$ to $D$ in equilibrium; the sucker's payoff must be sufficiently large for punishment to be incentive-compatible. The new conditions involve temptation payoff and productivity parameter. Productivities cannot be too different or productive players would avoid punishing in asymmetric meetings. The proof follows. 


\subsection{Contagious punishment}

When everyone follows the strategy in Definition 1, partition the population into $N-k$ cooperators and $k \in \kappa:=(1, \ldots, N)^{\top}$ defectors. Cooperators follow equilibrium play; Defectors only play $D$. The economy is off-equilibrium if $k \geq 2 ; k=1$ denotes when player $i$ moves off-equilibrium in a match $\theta_{i} \geq \theta_{-i}$. Indeed, player $i$ has no incentive to deviate to $C$ from $D$ if $\theta_{i}<\theta_{-i}$.

The $N \times N$ upper-triangular transition matrix

$$
\mathcal{Q}_{N}=\left(\begin{array}{ccccccccc}
Q_{11} & Q_{12} & 0 & 0 & 0 & 0 & \ldots & 0 & 0 \\
0 & Q_{22} & Q_{23} & Q_{24} & 0 & 0 & \ldots & 0 & 0 \\
0 & 0 & Q_{33} & Q_{34} & Q_{35} & Q_{36} & \ldots & 0 & 0 \\
\vdots & \vdots & \vdots & \vdots & \vdots & \vdots & \ldots & \vdots & \vdots \\
0 & 0 & 0 & 0 & 0 & 0 & \ldots & Q_{N-1, N-1} & Q_{N-1, N} \\
0 & 0 & 0 & 0 & 0 & 0 & \ldots & 0 & 1
\end{array}\right)
$$

describes the contagious punishment process.

The first row applies if $i$ moves off-equilibrium when $\theta_{i} \geq \theta_{-i}$, with probability $Q_{12}=1-q(1-q)$. This triggers contagious punishment, gradually bringing the economy to full defection. $Q_{11}=(1-q) q$ is the probability that $\theta_{i}<\theta_{-i}$, so no punishment is triggered.

In the second row there are two defectors $i=\ell, m$. The number of defectors doubles with probability $Q_{24}$, if they both are in mixed matches where $\theta_{i} \geq \theta_{-i}$. If the defectors meet each other or are in matches where $\theta_{i}<\theta_{-i}$, then the number of defectors does not increase (with probability $Q_{22}$ ). If only one defector $i=\ell, m$ is in a mixed match with $\theta_{i} \geq \theta_{-i}$, then there is only one additional defector, with probability $Q_{23}$.

Not all mixed matches - cooperator-defector matches - contribute to spread punishment since $D$ is part of equilibrium play. This is the central difference with homogeneous economies Kandori (1992). When cooperator $i$ meets defector $-i$ and $\theta_{i}>\theta_{-i}, i$ will not start defecting since $-i$ follows equilibrium play. This match occurs with probability $q(1-q)$, so, if there are $j$ mixed matches, contagion occurs in $n<j$ of those with probability $\left(\begin{array}{l}j \\ n\end{array}\right)[1-q(1-q)]^{n}[q(1-q)]^{j-n}$. 
A transition from $k \geq 2$ to $k^{\prime} \geq k$ defectors occurs with probability

$$
Q_{k k^{\prime}}(N):=\sum_{j=k^{\prime}-k}^{\min (k, N-k)} \lambda_{k j}\left(\begin{array}{c}
j \\
k^{\prime}-k
\end{array}\right)[1-q(1-q)]^{k^{\prime}-k}[q(1-q)]^{j-k^{\prime}+k},
$$

where $N$ is omitted in $\mathcal{Q}_{N}$. The probability of $j$ mixed matches

$$
\lambda_{k j}:=\frac{j !\left(\begin{array}{c}
k \\
j
\end{array}\right)\left(\begin{array}{c}
N-k \\
j
\end{array}\right)(k-j-1) ! !(N-k-j-1) ! !}{(N-1) ! !},
$$

is the number of such pairings divided by the number $(N-1)$ !! of possible pairings, where

$$
j \in J_{k}:= \begin{cases}\{0,2,4, \ldots, \min (k, N-k)\} & \text { if } k=\text { even } \\ \{1,3,5, \ldots, \min (k, N-k)\} & \text { if } k=\text { odd }\end{cases}
$$

\subsection{Off-equilibrium continuation payoffs}

Let player $i$ be one of $k \geq 1$ defectors. She meets one of $N-k$ cooperators with probability $\sigma_{k}:=\frac{N-k}{N-1}$. Let $\sigma=\left(\sigma_{1}, \ldots, \sigma_{N-1}, 0\right)^{\top}$ and $e_{k}$ be the $N$-dimensional column vector with 1 in the $k^{s t}$ position and 0 everywhere else.

From Camera and Gioffré (2014, Theorem 2), the rate at which a defector expects to meet cooperators in the continuation game is

$$
\phi_{k}:=(1-\beta) e_{k}^{\top}\left(I-\beta \mathcal{Q}_{N}\right)^{-1} \sigma, \quad k \geq 1,
$$

where $\lim _{\beta \rightarrow 1^{-}} \frac{\phi_{k}}{1-\beta}<\infty$.

The beginning-of-period payoff to defector $i$ is

$$
v_{k}=\sigma_{k} \pi+\beta \sum_{k^{\prime}=k}^{N} Q_{k k^{\prime}} v_{k^{\prime}}, \quad \text { for } k \geq 1,
$$

where

$$
\pi:=(c+g)\left[q \alpha+(1-q)^{2}\right]
$$

denotes earnings expected ex-ante in a mixed match.

Letting $\mathbf{v}:=\left(v_{1}, \ldots, v_{N}\right)^{\top}$, we have

$$
\mathbf{v}=\sigma \pi+\beta \mathcal{Q}_{N} \mathbf{v} \quad \Rightarrow \quad \mathbf{v}=\left(I-\beta \mathcal{Q}_{N}\right)^{-1} \sigma \pi
$$


and we have $v_{k+1} \leq v_{k}$ with

$$
v_{k}=e_{k}^{\top}\left(I-\beta \mathcal{Q}_{N}\right)^{-1} \sigma \pi=\frac{\pi \phi_{k}}{1-\beta} .
$$

\subsection{Equilibrium deviations}

Deviating to $D$ in symmetric matches $\theta_{i}=\theta_{-i}=\theta$ is suboptimal if

$$
\theta c+\beta \frac{\pi^{*}}{1-\beta} \geq \theta(c+g)+\beta v_{2}
$$

Here $\theta_{i}=\theta_{-i}=\alpha$ represents the most stringent case, and we have

$$
\alpha c+\beta \frac{\pi^{*}}{1-\beta}-\alpha(c+g)-\beta v_{2}=-\alpha g+\frac{\beta \pi^{*}}{1-\beta}-\frac{\beta \phi_{2} \pi}{1-\beta} .
$$

Since $\lim _{\beta \rightarrow 1} \frac{\phi_{k}}{1-\beta}<\infty$ for all $k \geq 1$, and $\pi^{*}>0$, by continuity there exists a $\beta_{s}^{*} \in(0,1)$ such that $(6)$ holds for $\beta \in\left[\beta_{s}^{*}, 1\right)$ and $\theta=1, \alpha$.

Deviating to $D$ in asymmetric matches $\theta_{i}>\theta_{-i}$ is suboptimal for $i$ if

$$
-l+\beta \frac{\pi^{*}}{1-\beta} \geq \beta v_{2}
$$

or equivalently

$$
\frac{\beta \pi^{*}}{1-\beta}-\frac{\beta \phi_{2} \pi}{1-\beta} \geq l
$$

Using again $\lim _{\beta \rightarrow 1} \frac{\phi_{k}}{1-\beta}<\infty$ and $\pi^{*}>0$, by continuity there exists $\beta_{a}^{*} \in(0,1)$ such that (8) holds for all $\beta \in\left[\beta_{a}^{*}, 1\right)$. Letting $\beta^{*}:=\max \left(\beta_{a}^{*}, \beta_{s}^{*}\right)$ equilibrium deviations are suboptimal if $\beta \in\left[\beta^{*}, 1\right)$.

\subsection{Off-equilibrium deviations}

When $k \geq 2$ a deviation occurs when defector $i$ chooses $C$ instead of $D$, in a match where $\theta_{i} \geq \theta_{-i}$.

Asymmetric matches. If $\theta_{i}>\theta_{-i}$, defector $i$ surely earns $-l$ since the other selects $D$. The deviation slows down contagion if $-i$ is a cooperator, with probability $\sigma_{k}$. The transition matrix $\mathcal{Q}_{N-2}$ with elements $Q_{k k^{\prime}}(N-2)$ describes contagion in all other meetings. 
The payoff to defector $i$ from deviating is

$$
-l+\overbrace{\sigma_{k} \beta \sum_{k^{\prime}=k-1}^{N-2} Q_{k-1, k^{\prime}}(N-2) v_{k^{\prime}+1}}^{i \text { meets a cooperator }}+\overbrace{\left(1-\sigma_{k}\right) \beta \sum_{k^{\prime}=k-2}^{N-2} Q_{k-2, k^{\prime}}(N-2) v_{k^{\prime}+2}}^{i \text { meets a defector }}
$$

Continuation payoffs depend on whether $-i$ is a cooperator or not. If she is, deviating limits future defectors to $k^{\prime}+1$ instead of $k^{\prime}+2$. The transition probabilities $Q_{k-1, k^{\prime}}(N-2)$ account for this by considering all matchings among $k-1$ defectors ( $k$ defectors excluding $i$ ) and $N-k-1$ cooperators $(N-k$ cooperators excluding $-i$ ). Here, deviating raises $i$ 's continuation payoff, because $v_{k^{\prime}}$ falls in $k^{\prime}$. If $i$ meets a defector no such benefit exists; the transition probabilities $Q_{k-2, k^{\prime}}(N-2)$ account for this meeting by considering all matchings among $k-2$ defectors $(k$ defectors excluding $i$ and $-i$ ) and $N-k$ cooperators. Deviating to $C$ (instead of $D)$ is suboptimal if

$$
\begin{aligned}
& -l+\sigma_{k} \beta \sum_{k^{\prime}=k-1}^{N-2} Q_{k-1, k^{\prime}}(N-2) v_{k^{\prime}+1}+\left(1-\sigma_{k}\right) \beta \sum_{k^{\prime}=k-2}^{N-2} Q_{k-2, k^{\prime}}(N-2) v_{k^{\prime}+2} \\
& \leq \sigma_{k} \beta \sum_{k^{\prime}=k-1}^{N-2} Q_{k-1, k^{\prime}}(N-2) v_{k^{\prime}+2}+\left(1-\sigma_{k}\right) \beta \sum_{k^{\prime}=k-2}^{N-2} Q_{k-2, k^{\prime}}(N-2) v_{k^{\prime}+2},
\end{aligned}
$$

that is

$$
\sigma_{k} \beta \sum_{k^{\prime}=k-1}^{N-2} Q_{k-1, k^{\prime}}(N-2)\left(v_{k^{\prime}+1}-v_{k^{\prime}+2}\right) \leq l, \quad \text { for } k \geq 2 .
$$

Using $v_{k}$ :

$$
\sigma_{k} \pi \beta \sum_{k^{\prime}=k-1}^{N-2} Q_{k-1, k^{\prime}}(N-2) \frac{\phi_{k^{\prime}+1}-\phi_{k^{\prime}+2}}{1-\beta} \leq l, \quad \text { for } k \geq 2 .
$$

Symmetric matches. If $\theta_{i}=\theta_{-i}$, the expected payoff to defector $i$ who deviates to $C$ from $D$ when $k \geq 2$ is

$$
\sigma_{\left[\theta_{i} c+\beta \sum_{k^{\prime}=k-1}^{N-2} Q_{k-1, k^{\prime}}(N-2) v_{k^{\prime}+1}\right]}^{i \text { meets a cooperator }}+\left(1-\sigma_{k}\right) \overbrace{\left[-l+\beta \sum_{k^{\prime}=k-2}^{N-2} Q_{k-2, k^{\prime}}(N-2) v_{k^{\prime}+2}\right]}^{i \text { meets a defector }} .
$$


Deviating is suboptimal if

$$
\begin{aligned}
& \sigma_{k} \theta_{i} c+\left(1-\sigma_{k}\right)(-l)+\sigma_{k} \beta \sum_{k^{\prime}=k-1}^{N-2} Q_{k-1, k^{\prime}}(N-2) v_{k^{\prime}+1}+\left(1-\sigma_{k}\right) \beta \sum_{k^{\prime}=k-2}^{N-2} Q_{k-2, k^{\prime}}(N-2) v_{k^{\prime}+2} \\
& \leq \sigma_{k} \theta_{i}(c+g)+\sigma_{k} \beta \sum_{k^{\prime}=k-1}^{N-2} Q_{k-1, k^{\prime}}(N-2) v_{k^{\prime}+2}+\left(1-\sigma_{k}\right) \beta \sum_{k^{\prime}=k-2}^{N-2} Q_{k-2, k^{\prime}}(N-2) v_{k^{\prime}+2},
\end{aligned}
$$

yielding

$$
\sigma_{k} \beta \sum_{k^{\prime}=k-1}^{N-2} Q_{k-1, k^{\prime}}(N-2)\left(v_{k^{\prime}+1}-v_{k^{\prime}+2}\right) \leq \sigma_{k} \theta_{i} g+\left(1-\sigma_{k}\right) l,
$$

or equivalently

$$
\sigma_{k} \pi \beta \sum_{k^{\prime}=k-1}^{N-2} Q_{k-1, k^{\prime}}(N-2) \frac{\phi_{k^{\prime}+1}-\phi_{k^{\prime}+2}}{1-\beta} \leq \sigma_{k} \theta_{i} g+\left(1-\sigma_{k}\right) l,
$$

most stringent when $\theta_{i}=1$.

From Camera and Gioffré (2014, Theorem 2), the most stringent case for (10) and (11) is $k=2$. A sufficient condition to avoid off-equilibrium deviations is

$$
\pi \gamma_{q} \leq \min (g, l)
$$

where

$$
\gamma_{q}:=\sup _{\beta \in(0,1)} \sum_{k^{\prime}=1}^{N-2} Q_{1, k^{\prime}}(N-2) \beta \frac{\phi_{k^{\prime}+1}-\phi_{k^{\prime}+2}}{1-\beta} .
$$

Lemma 1. For all $q \in[0,1]$ and $n \in \mathbb{N}_{+}$, the function $\gamma_{q} \in(0,1)$.

The proof is in Appendix. Using the definition of $\pi$, (12) becomes

$$
\gamma_{q}\left[q \alpha+\left(1-q^{2}\right)\right] \leq \frac{\min (g, l)}{c+g}
$$

From Lemma $1 \gamma_{q}<1$, so if $g<l$, a necessary condition for (12) is

$$
\alpha<\alpha_{q}:=\frac{1-\gamma_{q}(1-q)^{2}}{\gamma_{q} q} .
$$

Since $l<\alpha g-c$ two cases arise.

1. $l \leq g: l<\alpha g-c$ implies $\alpha>\frac{l+c}{g}$, which holds if $\alpha>\frac{g+c}{g}$.

2. $g<l<\alpha g-c$ : we need $g<\alpha g-c$, implying $\alpha>\frac{c+g}{g}$.

Hence, letting $\underline{\alpha}:=\frac{c+g}{g}$, if $c / g$ is sufficiently small, then there exists $\alpha \in$ 
$\left(\underline{\alpha}, \alpha_{q}\right)$ ensuring that deviating off-equilibrium is suboptimal.

\section{References}

Blonski, P. and G. Spagnolo. 2015. Prisoner's other dilemma. International Journal of Game Theory 44, 61-81

Camera, G., Gioffré, A., 2014. A tractable analysis of contagious equilibria. Journal of Mathematical Economics 50, 290-300.

Ellison, G., 1994. Cooperation in the prisoner's dilemma with anonymous random matching. Review of Economic Studies, 61, 567-88.

Fudenberg, D. and Maskin, E. 1986. The Folk Theorem in Repeated Games with Discounting or with Incomplete Information. Econometrica 50, 533-554.

Kandori, M., 1992. Social norms and community enforcement. Review of Economic Studies, 59, 63-80.

Rapoport, A. and Chammah, A. M. 1965. Prisoner's Dilemma: A Study in Conflict and Cooperation. University of Michigan Press, Ann Arbor.

Rubinstein, A. (1979), Equilibrium in Supergames with the Overtaking Criterion. Journal of Economic Theory, 21, 1-9. 


\section{Appendix: Proof of Lemma 1}

The cases $q=0$ and $q=1$ are in Camera and Gioffré $(2014)$. For $q \in(0,1)$

$$
\begin{aligned}
\gamma_{q}(\beta) & :=\sum_{k^{\prime}=1}^{N-2} Q_{1, k^{\prime}}(N-2) \beta \frac{\phi_{k^{\prime}+1}-\phi_{k^{\prime}+2}}{1-\beta} \\
& =q(1-q) \beta \frac{\phi_{2}-\phi_{3}}{1-\beta}+[1-q(1-q)] \beta \frac{\phi_{3}-\phi_{4}}{1-\beta} .
\end{aligned}
$$

For any $\beta \in(0,1)$ and $\forall k \geq 1$ we have $\phi_{k}>\phi_{k+1}$ (Camera and Gioffré, 2014, Theorem 2), so $\gamma_{q}(\beta)>0$. To show that $\gamma_{q}<1$, the payoff $v_{2}$ is

$$
\begin{aligned}
v_{2} & =\sigma_{2}\left\{\pi+\beta[\overbrace{(1-q(1-q)) \sum_{k^{\prime}=1}^{N-2} Q_{1, k^{\prime}}(N-2) v_{k^{\prime}+2}}^{i \text { transmits the defection }}+\overbrace{\left.q(1-q) \sum_{k^{\prime}=1}^{N-2} Q_{1, k^{\prime}}(N-2) v_{k^{\prime}+1}\right]}^{i \text { does not transmit the defection }}\}\right. \\
& +\overbrace{\left(1-\sigma_{2}\right)\left[d+\beta v_{2}\right]}^{i \text { meets the other defector }},
\end{aligned}
$$

or, equivalently,

$$
\begin{aligned}
v_{2} & =\sigma_{2}(\pi-d)+d+\sigma_{2} \beta[1-q(1-q)]\left\{q(1-q) v_{3}+[1-q(1-q)] v_{4}\right\} \\
& +\sigma_{2} \beta q(1-q)\left\{q(1-q) v_{2}+[1-q(1-q)] v_{3}\right\}+\left(1-\sigma_{2}\right) \beta v_{2} .
\end{aligned}
$$

Rearranging

$\sigma_{2}\left[1-q^{2}(1-q)^{2}\right] \beta\left(v_{2}-v_{3}\right)=\sigma_{2}(\pi-d)+d-v_{2}(1-\beta)-\sigma_{2} \beta[1-q(1-q)]^{2}\left(v_{3}-v_{4}\right)$,

and using (5)

$$
\beta \frac{\phi_{2}-\phi_{3}}{1-\beta}=\frac{1}{1-q^{2}(1-q)^{2}}\left(1-\frac{\phi_{2}}{\sigma_{2}}\right)-\frac{[1-q(1-q)]^{2}}{1-q^{2}(1-q)^{2}} \beta \frac{\phi_{3}-\phi_{4}}{1-\beta} .
$$

Using the definition of $\gamma_{q}(\beta)$ :

$$
\gamma_{q}(\beta)=\frac{q(1-q)}{1-q^{2}(1-q)^{2}}\left(1-\frac{\phi_{2}}{\sigma_{2}}\right)+\frac{[1-q(1-q)]^{2}}{1-q^{2}(1-q)^{2}} \beta \frac{\phi_{3}-\phi_{4}}{1-\beta} .
$$

and since $\phi_{3}-\phi_{4} \leq \phi_{2}-\phi_{3}$ (see Camera and Gioffré, 2014, Theorem 2) we have

$$
\gamma_{q}(\beta) \leq \frac{q(1-q)}{1-q^{2}(1-q)^{2}}\left(1-\frac{\phi_{2}}{\sigma_{2}}\right)+\frac{[1-q(1-q)]^{2}}{1-q^{2}(1-q)^{2}} \beta \frac{\phi_{2}-\phi_{3}}{1-\beta} .
$$


Again using (13):

$\gamma_{q}(\beta) \leq \overbrace{\frac{1+q^{2}(1-q)^{2}}{[1+q(1-q)]\left[1-q^{2}(1-q)^{2}\right]}}^{\leq 1 \forall q \in[0,1]} \overbrace{\left(1-\frac{\phi_{2}}{\sigma_{2}}\right)}^{<1}-\overbrace{\frac{[1-q(1-q)]^{4}}{\left[1-q^{2}(1-q)^{2}\right]^{2}} \beta \frac{\phi_{3}-\phi_{4}}{1-\beta}}^{\geq 0}<1$

where we used $\phi_{2}<\sigma_{2}$, as proved in Camera and Gioffré (2014, Theorem 2).

Taking the supremum of $\gamma_{q}(\beta)$ concludes the proof. 